\title{
Hybrid analysis method using CFD and analytical solutions on fluid elastic vibration of circular cylinder arrays
}

\author{
K. Fujita ${ }^{1,2} \&$ T. Wakita ${ }^{3}$ \\ ${ }^{1}$ Institute of Nuclear Safety System Inc., Japan \\ ${ }^{2}$ Fujita Mechanical Dynamics Laboratory, Japan \\ ${ }^{3}$ Kobe Steel Ltd., Japan
}

\begin{abstract}
In this paper, the hybrid method using the computational fluid dynamics (CFD) and the analytical solution on the fluid elastic vibration of circular cylinder arrays is proposed. The fluid forces acting on cylinder arrays are calculated as the influence coefficients which are obtained by the sinusoidal excitation on a cylinder in a small-scale of cylinder arrays subjected to a cross flow in the CFD simulation. Applying these fluid forces to a large-scale of cylinder arrays, the entire equations of motion can be derived for stability analysis. Comparing the direct simulation analysis based on CFD, the proposed hybrid method is found to be useful for evaluating the critical velocity of the fluid elastic vibration with less computational time. In addition, the present method is also very convenient for grasping the physical meaning on the fluid elastic vibration behavior, such as evaluating which vibration mode becomes unstable fastest among a group of complex eigenvalues generated by the coupled vibration among many circular cylinders in a fluid.

Keywords: fluid elastic vibration, circular cylinder arrays, cross flow induced vibration, CFD simulation, stability analysis.
\end{abstract}

\section{Introduction}

Many studies have been already performed on the mechanism of the fluid elastic vibration of circular cylinder arrays occurred in heat exchangers and stream generators, experimentally and analytically. Conners, Hartlen, Gorman, Zdravkovich, S. S. Chen and Ishigai et al. can be listed up first as the contributors 
to developing the technical progress [1]. However, as there are too many subjects concerning the fluid elastic vibration of cylinder arrays, the necessity of further investigations is considered to remain.

In this paper, the hybrid method using the computational fluid dynamics (CFD) and the analytical solution for the fluid elastic vibration of circular cylinder arrays is proposed. First, the fluid forces acting on cylinder arrays are calculated as the influence coefficients which are obtained by the sinusoidal excitation on a cylinder in a small-scale of cylinder arrays subjected to a cross flow in CFD simulation. Applying the concept of cyclic symmetry on those fluid forces of a basic cylinder arrays, the fluid forces for a large-scale of cylinder arrays is obtained following the proposed algorithm. Thus, the equations of motion on the stability analysis for a large scale of cylinder arrays can be derived by using these fluid forces. The validity of the proposed hybrid method is confirmed by comparing the present results with the experimental results reported in the literature.

\section{Hybrid analysis using CFD and analytical solution}

\subsection{Modeling for CFD simulation}

Figure 1 shows the dimensions of nine cylinders for CFD simulations. The cylinder No. 1 is elastic and can oscillate. The cylinders No. 2 to No. 9 are rigid. Figure 2 shows the mesh of the finite element for CFD simulation. The mesh in the vicinity of circular cylinders are divided finely, and the length of the entrance passage is sufficiently long compared with the cylinder diameter in order to stabilize the flow in numerical calculation.

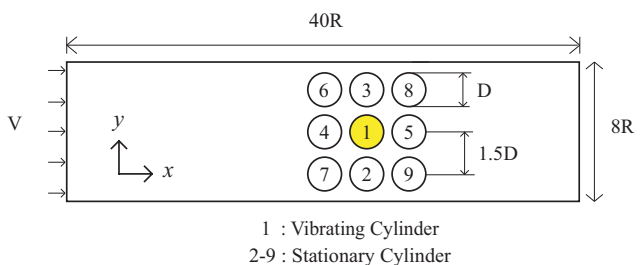

Figure 1: Multi-cylinder model.

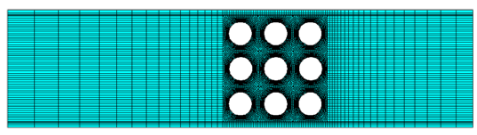

Figure 2: Mesh for fluid calculation.

\subsection{Calculation of unsteady fluid forces}

The cylinder No. 1 shown in Fig. 1 is excited sinusoidally. The unsteady fluid forces can be obtained by integrating the unsteady fluid pressure acting on the outer surface of cylinders when the cylinder No.1 is excited taking the fluid velocity as a parameter. The following unsteady force, which consists of a fluid inertia force, 
a fluid damping force and a fluid stiffness force, is obtained as reported in Tanaka and Takahara [2]:

$$
F=\frac{\pi \rho d^{2}}{4} C_{m} \ddot{X}+\frac{\rho d V}{2} C_{D} \dot{X}+\frac{\rho V^{2}}{2} C_{K} X,
$$

where $\rho$ is the fluid density, $d$ the cylinder diameter, and $C_{m}, C_{D}$ and $C_{K}$ are the coefficients of added mass, added damping and added stiffness, respectively. Moreover, $X$ denotes the displacement of a cylinder, and the derivative of time is denoted by an over-dot. Assuming the stationary vibrational state, let us express $X$ as follows:

$$
X=X_{0} \cos \left(\omega_{E} t\right)
$$

where $X_{0}$ is the vibration amplitude. Furthermore, when we introduce the reduced velocity $V_{R}\left(=V / f_{E} d\right)$, eqn. (1) can be represented as follows:

$$
F=\frac{\rho V^{2} X_{0}}{2}\left[\left(\frac{-2 \pi^{3}}{V_{R}^{2}} C_{m}+C_{K}\right) \cos \left(\omega_{E} t\right)+\frac{2 \pi}{V_{R}} C_{D} \sin \left(\omega_{E} t\right)\right],
$$

where $f_{E}$ is the exciting frequency, and $f_{E}=\omega_{E} / 2 \pi$. On the other hand, as the unsteady force is proportional to the amplitude of the exciting displacement, it can be expressed by the following equation considering the phase lag $\phi$ between the exciting displacement and the calculated fluid force:

$$
F=-\frac{1}{2} \rho V^{2} C X_{0} \cos \left(\omega_{E} t+\phi\right),
$$

where $\mathrm{C}$ is the constant coefficient. Expanding eqn. (4) to the Fourier series, the following equation is obtained:

$$
C_{D}=-\frac{V_{R}}{2 \pi} C \sin \phi, \quad C_{K}=-C \cos \phi+2 \frac{\pi^{3}}{V_{R}^{2}} C_{m},
$$

where $C_{m}$ can be calculated in a still fluid assuming it is not dependent on the fluid velocity.

When we calculate the unsteady force $F(t)$ concretely, the ALE (Arbitrary Lagrangian-Eulerian) method is applied. Integrating the fluid pressure acting on the outer surface of the cylinders, $C_{D}$ and $C_{K}$ can be determined by eqn. (5) using $C_{m}$ which is already obtained in a still fluid. The fluid analysis and the structure analysis are performed alternatively, and then the time history waves of fluid forces are obtained step by step.

\subsection{Formation of unsteady fluid forces acting on cylinders}

When the multi-cylinders are subjected to a cross flow, the unsteady force acting on the arbitrary oscillating cylinder is influenced by itself and the adjacent oscillating 
cylinders. For example, for an $\mathrm{n} \times \mathrm{n}$ cylinder array, an arbitrary cylinder interacts with the surrounding adjacent cylinders most strongly, and with the surrounding far cylinders more weakly. In this paper, let us define the nine cylinders array shown in Fig. 1 as a basic cylinder array. Assuming unsteady fluid forces acting on the cylinder No.1 to be expressed as the function of displacements of the top and bottom cylinders, the right and left cylinders as shown in Fig. 1. The total fluid forces acting on the cylinder No.1, $F_{X}, F_{Y}$ can be given as follows:

$$
\begin{aligned}
& F_{X}=\frac{\rho V^{2}}{2} \sum_{k=1}^{5}\left(C_{x k x} X_{k}+C_{x k y} Y_{k}\right), \\
& F_{Y}=\frac{\rho V^{2}}{2} \sum_{k=1}^{5}\left(C_{y k x} X_{k}+C_{y k y} Y_{k}\right),
\end{aligned}
$$

where the subscripts of coefficients $C_{x k x} \sim C_{y k y}$ show the direction of the acting fluid, the number of a cylinder and the direction of oscillation, respectively, from the left to the right. Besides, the influences of the oblique adjacent cylinders are neglected for simplification.

\subsection{Equations of motion by hybrid method using CFD and analytical solution}

The coupled equations of motion for the cylinder array using the above-mentioned fluid forces acting on the cylinder can be derived as follows:

$$
\sum_{j=1}^{n}\left(m_{i j} \ddot{X}_{j}+D_{i j} \dot{X}_{j}+k_{i j} X_{j}\right)=0 \quad(i=1,2, \ldots n),
$$

where $\mathrm{N}$ is the total number of cylinders, and $X_{j}$ is the displacement of the $\mathrm{j}$-th cylinder. The coefficients are given by the following equations:

$$
\begin{gathered}
m_{i j}=\left(\delta_{c i j}+\frac{\pi C_{m i j}}{4 \mu_{i}}\right), \\
D_{i j}=\left(\frac{\omega_{i} \delta_{i}^{s}}{\pi} \delta_{c i j}+\frac{C_{D i j} V}{2 \mu_{i} d}\right), \\
k_{i j}=\left(\omega_{i}^{2} \delta_{c i j}+\frac{C_{K i j} V^{2}}{2 \mu_{i} d^{2}}\right),
\end{gathered}
$$

where $\mu_{i}=m_{i} / \rho d^{2}$, and $\delta_{i}^{s}$ is the logarithmic decrement, $\delta_{c i j}$ the Kronecker's delta function. Rewriting the above-mentioned equations of motion into the 
matrices form, the following equations are obtained for the complex eigenvalue analysis:

$$
\begin{gathered}
\{\dot{X}\}=[B]\{X\}, \quad\{X\}=\left\{x_{i}, y_{i}, \dot{x}_{i}, \dot{y}_{i}\right\}^{T}, \\
{[B]=\left[\begin{array}{cc}
0 & I \\
-T & -S
\end{array}\right],} \\
{[T]=\left[\frac{\Omega_{i}^{2}}{\beta_{i}^{2}} \delta_{c} i j+\frac{V_{R}^{2}}{8 \pi^{2} \mu_{i}}\left[C_{K} i j\right]\right]\left[\delta_{c} i j+\frac{\pi}{4 \mu_{i}}\left[C_{m} i j\right]\right]^{-1},} \\
{[S]=\left[\frac{\delta_{i}^{s} \Omega_{i}}{\beta_{i} \pi} \delta_{c} i j+\frac{V_{R}}{4 \pi \mu_{i}}\left[C_{D} i j\right]\right]\left[\delta_{c} i j+\frac{\pi}{4 \mu_{i}}\left[C_{m} i j\right]\right]^{-1},}
\end{gathered}
$$

where $\Omega_{i}=\omega_{i} / \omega_{0}, \beta_{i}=\omega / \omega_{0}, V_{R}=2 \pi V /(\omega d)$, and $\omega_{i}$ is the natural circular frequency of the cylinder, $\omega_{0}$ is the initial frequency and $\omega$ is the natural circular frequency of a cylinder in a still fluid.

The complex eigenvalues and eigenmodes can be obtained by complex-analysis of eqn. (12). The real part of the eigenvalues: $\lambda_{R j}>0$ and the imaginary part of the eigenvalues: $\lambda_{I j} \neq 0$ indicates a dynamic instability(flutter), and $\lambda_{R j}>0, \lambda_{I j}=0$ indicates a static instability(divergence).

\subsection{Expansion for $\mathbf{n} \times \mathbf{n}$ cylinder array}

As shown in Fig. 1, we calculate the influence coefficients of fluid forces setting the $3 \times 3$ cylinder array as a basic cylinder array for deriving the unsteady fluid forces. Superposing these influence coefficients which are obtained by using the basic $3 \times 3$ cylinder array based on the concept of cyclic symmetry, the equation of motion for an $\mathrm{n} \times \mathrm{n}$ cylinder array can be derived, and the unstable analysis of the critical velocity can be performed.

\section{Numerical analysis and considerations}

The results obtained in this study were compared with the experimental results reported by Weaver and Abd-Rabbo [3] and Tanaka and Takahara [2] in order to prove the validity of the hybrid method proposed herein. As shown in Table 1, the simulations were performed for the same cylinder array with the same dimensions (a $3 \times 3$ in-line cylinder array). These models will be referred to as either the "Weaver model" or the "Tanaka model" below. 


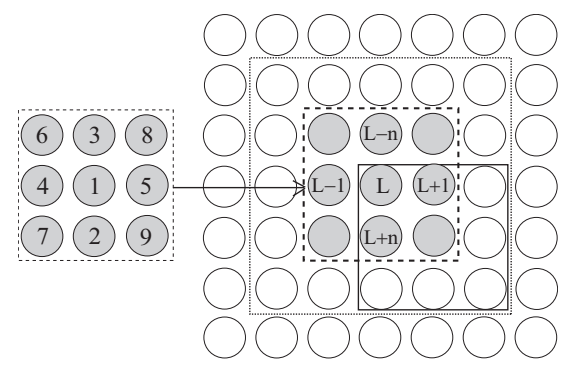

Figure 3: Expansion of calculation algorithm to $\mathrm{n} \times \mathrm{n}$ cylinder array.

Table 1: Parameters of analysis.

\begin{tabular}{lcc}
\hline & Weaver & Tanaka \\
\hline Pitch ratio & 1.5 & 1.3 \\
\hline $\begin{array}{l}\text { Mass-Damping } \\
\text { Parameter }\left(M \delta / \rho d^{2}\right)\end{array}$ & 0.07 & 0.3 \\
\hline $\begin{array}{l}\text { Logarithmic } \\
\text { damping }(\delta)\end{array}$ & 0.037 & 0.141 \\
\hline $\begin{array}{l}\text { Natural frequency } \\
\text { (Elastic cylinder) }\end{array}$ & 28 & 34 \\
\hline
\end{tabular}

\subsection{CFD simulation results}

Figure 4 presents the changes in fluid pressure distribution over one period of exciting frequency for the Weaver model. This figure illustrates the fluid pressures contour when the cylinder No.1 is excited by $3 \mathrm{~Hz}$ harmonic vibration with the amplitude of ten-percent of diameter under the reduced velocity $V_{R}=2.4$.

\subsection{Comparison of present analysis and experiments by Weaver et al.}

Even for the two-dimensional analysis of the $3 \times 3$ cylinder array, 18 eigenfrequencies exist due to fluid-structure interaction and a considerable range between the highest and lowest eigenfrequencies is formed. There is also a great variety in the vibration modes. Figure 5 shows the root-locus diagram (Argand diagram) obtained by the complex eigenvalue analysis of the Weaver model. Figure 6 indicates the relationships between the reduced velocity $V_{R}$ and the real part $\lambda_{R j}$ and imaginary part $\lambda_{I j}$. In this figure, there are 18 damping values in the real part and 18 natural frequencies in the imaginary part at the same arbitrary reduced velocity. When the real part of the eigenvalue crosses the horizontal axis to become positive, the eigenmode is judged to become unstable. On the other hand, the 


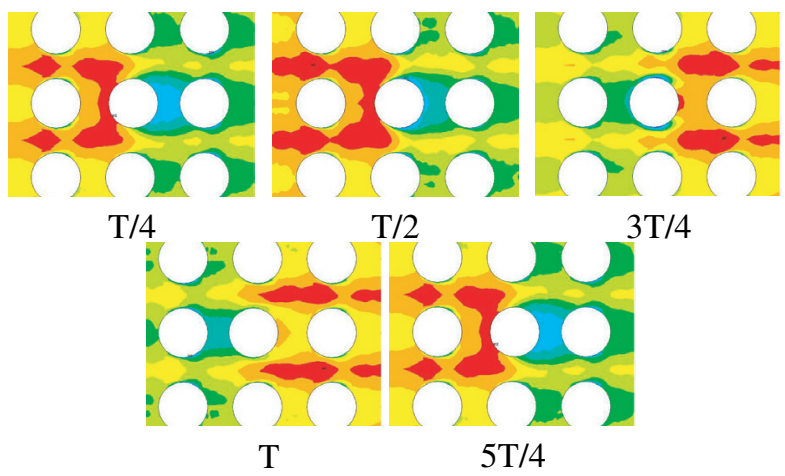

Figure 4: Pressure contour over one period, excited in the x-direction.

imaginary part which corresponds to the positive real part indicates the frequency at which the instability occurs among a wide range of eigenfrequencies. The $\bullet$ symbol in the figure denotes stable, while $\times$ denotes unstable.

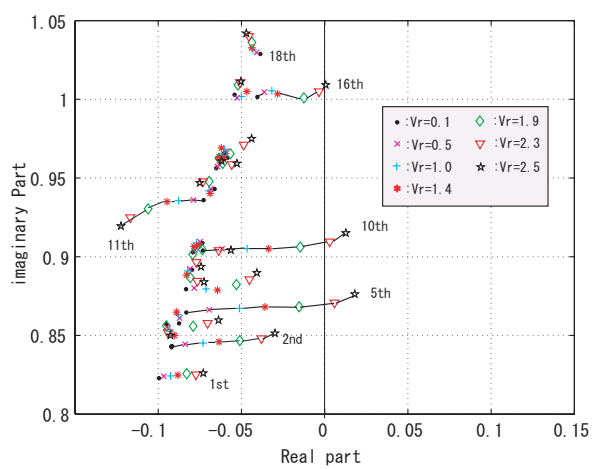

Figure 5: Root locus (Argand diagram), Weaver model $(3 \times 3)$ pitch ratio 1.5.

The critical velocity of $V_{C R}=2.45$ in the experiments by Weaver et al., and that obtained by the present hybrid method $V_{C R}=2.3$ are considered to be considerably similar. Being clear in the root-locus diagram, the lowest initial eigenmode of an array is not necessarily the one that first goes into instability. Figure 7 shows the vibration modes at the critical velocity. From this figure, the cylinders in the stable modes are found to vibrate in the same direction, like the heads of rice stalks in the wind. In contrast, the tenth mode which is unstable, exhibits a continual change in the relative displacement of cylinders. When any pair of neighboring cylinders approaches each other, the cylinders next to this pair tend to move away from the pair. This phenomenon is seen in both stream wise and transverse motions. The cylinder loci are elliptical. These results are consistent with the analytical assumption that the movement of the cylinders in different 


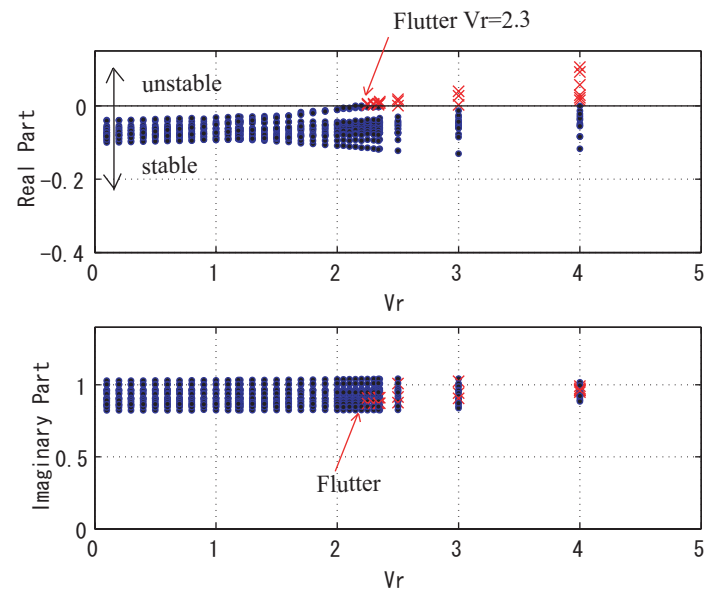

Figure 6: Relationship between real part and imaginary part of complex eigenvalue analysis and reduced velocity, Weaver model $(3 \times 3)$ pitch ratio 1.5 .

directions causes alternative narrowing and widening of the interstitial flow path and contributes to coupling between the fluid elastic forces and the elastic forces of cylinders. Observing the changes of the vibration mode-shape from in a still fluid to at critical flow velocity, it is revealed that the closer the velocity is to the critical value, the greater the elliptical motion.

\subsection{Comparison of present analysis with experiments by Tanaka et al.}

Comparing with the Weaver model with pitch ratio 1.5 , the same complex eigenvalue analysis is carried out on the Tanaka model with the pitch ratio 1.3. Figure 8 shows the changes in the real and imaginary parts of the eigenvalues with reduced velocity. The critical velocity $V_{C R}=2.5$ is found in this simulation in comparison to 2.7 in experiment by Tanaka et al.. Similar to the case of Weaver model, a higher eigenmode, not the lowest eigenmode, is found to start fluttering first. In contrast, for the 16th mode, which is unstable, the adjacent cylinders also exhibit violent change in relative position.

\subsection{Influence of number of cylinders on critical velocity}

The influence of the number of cylinders on the critical velocity was examined by increasing the number of cylinders beyond the basic $3 \times 3$ array. Figure 9 displays the results obtained for the arrays of $2 \times 2,3 \times 3,4 \times 4,5 \times 5,6 \times 6$ and $7 \times 7$ cylinders. In the $6 \times 6$ and larger arrays, the critical velocity is found to become roughly constant. The reported experimental studies have already indicated that the critical velocity decreases with the number of cylinders. 


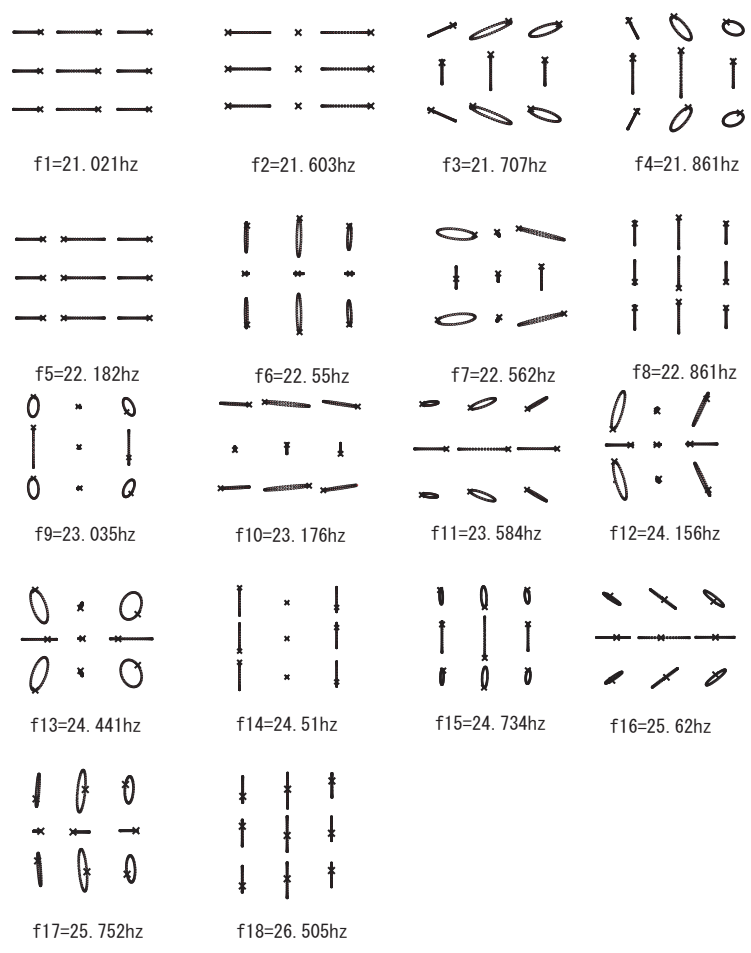

Figure 7: Vibration mode at $\operatorname{Vrcr}=2.3$, Weaver model $(3 \times 3)$, pitch ratio 1.5.
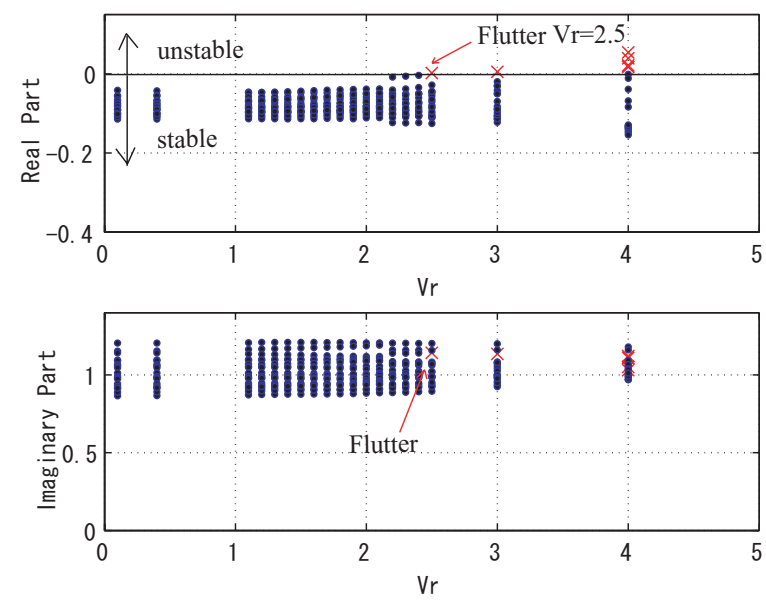

Figure 8: Relationship between real part and imaginary part of complex eigenvalue analysis and reduced velocity, Tanaka model $(3 \times 3)$ pitch ratio 1.3 . 


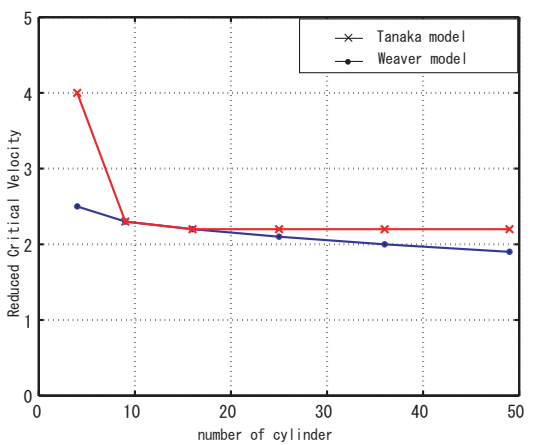

Figure 9: Effect of number of cylinders on critical reduced velocity.

\subsection{Critical velocities diagram of fluid elastic vibration}

Many experiments have been already performed as for determining the critical velocities of fluid elastic vibration as the design chart, and the results are summarized in line charts showing the stability limits. Figure 10 presents a comparison of those experiments and the results of the present hybrid simulation analysis. There is a good agreement between the previous experimental results and the present results.

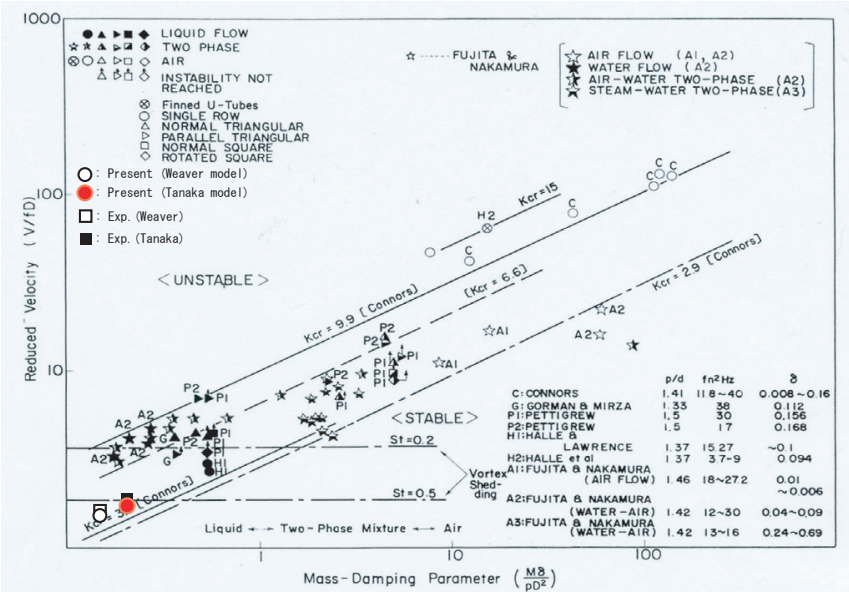

Figure 10: Critical velocity diagram of fluid elastic vibration. 


\section{Conclusions}

The proposed hybrid method combining CFD and analytical solutions have a good agreement with the previous experiments and is found to be practical for the stability analysis of the fluid elastic vibration of circular cylinder arrays. The vibration mode in which the adjacent cylinders exhibit the relative displacements and are out of phase is apt to be unstable. The proposed hybrid analytical method showed that the critical flow velocity decreases with increasing the number of cylinders. Comparing to direct CFD simulation, the proposed method also makes the parametric studies easy, and it is found to be more effective for providing the understanding of physical phenomena.

\section{References}

[1] Fujita,K., Flow-induced vibration and fluid-structure interaction in nuclear power plant components. Journal of Wind Engineering 37, pp. 577-590, 1988.

[2] Tanaka,H., Takahara,S., Fluid elastic vibration of tube array in cross flow. Journal of Sound and Vibration, 77, pp. 19-37, 1981.

[3] Weaver,D.S., Abd-Rabbo,A., A flow visualization study of a square array of tubes in water crossflow. ASME Journal of Fluid Engineering, 107, pp. 354363, 1985.

[4] Fujita,K., Wakita,T., Fluid elastic vibration analysis of circular cylinder arrays based on hybrid method using CFD and analytical solution. Proc. of The 8th Inter. Conf. on Flow Induced Vibration, FIV2004, Paris, 6-9 July, pp. 289-294, 2004. 